\title{
Voltage-gated calcium channels: Structure, permeability and inactivation properties
}

Pierre Charnet, Thierry Cens, Matthieu Rousset, Sophie Restituito, Claudine Menard and JeanBaptiste Thibaud $^{\text {a }}$

${ }^{1}$ IBMM UMR 5247, 1919 Route de Mende, Montpellier, France

\begin{abstract}
Our group has been interested in the structure and function of voltage-gated $\mathrm{Ca} 2+$ channels since many years. We have tried to combine molecular cloning, site-directed mutagenesis, heterologous expression, electrophysiological recordings and fluorescence imaging to identify the structures and molecular motions and understand the molecular mechanisms that are responsible for several biophysical properties of these channels. In this talk I will present you a brief overview of this work with a special emphasis on the voltage-dependent inactivation and channel selectivity toward divalent cations. Several references taken from studies on voltage-gated $\mathrm{K}+$ channels will introduce several parts of this presentation.
\end{abstract}

\section{Funding acknowledgment:}

ANR photoproteolyse (BLAN06-1_148568) and Bee-channels (ANR-13-BSV7-0010-03 )

${ }^{a}$ Corresponding author: pierre.charnet@,crbm.cnrs.fr 